\title{
Molecular characterization of patchouli (Pogostemon spp) germplasm
}

S.S. Sandes ${ }^{1}$, M.I. Zucchi², J.B. Pinheiro ${ }^{3}$, M.M. Bajay ${ }^{3}$, C.E.A. Batista ${ }^{3}$, F.A. Brito ${ }^{1}$, M.F. Arrigoni-Blank' ${ }^{1}$, S.V. Alvares-Carvalho ${ }^{1}$, R. Silva-Mann ${ }^{1}$ and A.F. Blank ${ }^{1}$

'Laboratório de Recursos Genéticos e Óleos Essenciais,

Departamento de Engenharia Agronômica, Universidade Federal de Sergipe,

São Cristóvão, SE, Brasil

'Laboratório de Biologia Molecular, Agência Paulista de Tecnologia dos Agronegócios, Polo Centro Sul, Piracicaba, SP, Brasil

${ }^{3}$ Laboratório de Diversidade Genética e Melhoramento, Departamento de Genética, Escola Superior de Agricultura "Luiz de Queiroz", Universidade de São Paulo,

Piracicaba, SP, Brasil

Corresponding author: A.F. Blank

E-mail: afblank@ufs.br

Genet. Mol. Res. 15 (1): gmr.15017458

Received August 17, 2015

Accepted October 29, 2015

Published February 19, 2016

DOI http://dx.doi.org/10.4238/gmr.15017458

ABSTRACT. Patchouli [Pogostemon cablin (Blanco) Benth.] is an aromatic, herbaceous plant belonging to the Lamiaceae family native to Southeast Asia. Its leaves produce an essential oil regularly used by the perfume and cosmetics industries. However, since patchouli from the Philippines and India were described and named Pogostemon patchouli, there has been a divergence in the identity of these species. The objective of the current study was to study the genetic diversity of patchouli accessions in the Active Germplasm Bank of Universidade Federal de Sergipe using microsatellite and inter simple sequence repeat markers. The results of both types of molecular markers showed that there are two well-defined clusters of accessions that harbor exclusive alleles. It was observed that these two clusters are genetically distant, suggesting that they belong to 
two different species. Based on the results, two accessions were classified as Pogostemon heyneanus and the remaining accessions were classified as $P$. cablin.

Key words: Pogostemon cablin; Pogostemon heyneanus; Microsatellites; ISSR

\section{INTRODUCTION}

Patchouli [Pogostemon cablin (Blanco) Benth.] is an herbaceous plant native to Southeast Asia and grown extensively in India, Malaysia, Philippines, Indonesia, and Singapore (Swamy et al., 2010). Patchouli essential oil is used by the perfume and cosmetic industries (Sugimura et al., 2005; Singh and Rao, 2009; Paul et al., 2010). Patchouli also has great relevance in traditional Chinese medicine, for which it is used to treat colds, vomiting, diarrhea, and as an antifungal treatment (Hsu et al., 2006; Xu et al., 2010).

Since patchouli from the Philippines ( $P$. cablin) and the Indian patchouli (Pogostemon heyneanus) were described and named Pogostemon patchouli, there has been a divergence in the identity of the two species (Murugan and Livingstone, 2010). According to these same authors, the names Pogostemon patchouly, P. patchouli, Pogostemon suavis, P. patchouli var. suavis, and Pogostemon javanicus refer to the same plant, $P$. cablin, which is the commercial patchouli. However, Oyen (1999) reported that $P$. cablin is a synonym of $P$. patchouly, whereas $P$. heyneanus is a synonym of $P$. patchouli. It is, thus, clear that even among researchers, there is disagreement regarding patchouli nomenclature and taxonomy. Therefore, it is necessary to use molecular tools to confirm the differences between these species, if they exist, to assist in the building and management of germplasm banks so that there are no issues in the identification of accessions of different species.

The use of molecular markers is typically efficient in studies identifying closely related individuals in germplasm banks. They provide important information in population genetics studies and in the management of natural resources because they are not influenced by environmental variables, as is the case for morpho-agronomical markers. Microsatellite markers, or single sequence repeats (SSR), are ubiquitous and abundant in the prokaryotic and eukaryotic genomes studied to data; they are present in coding and non-coding regions of the genome, and are generally characterized by a high degree of polymorphism compared with other molecular markers (Kelkar et al., 2010; Remya et al., 2010). PCR amplification of genomic DNA using a single primer that targets the SSR per se has resulted in a new type of analysis using inter-simple sequence repeats (ISSR) (Liu and Wendel, 2001). ISSR markers were developed by Zietkiewicz et al. (1994) and brought a new approach for fingerprinting, applicable to taxonomy and phylogenetic comparisons, as well as a mapping tool for a wide range of organisms (Zietkiewicz, et al., 1994).

The objective of the present study was to molecularly characterize the patchouli accessions of the Active Germplasm Bank of the Universidade Federal de Sergipe using SSR and ISSR.

\section{MATERIAL AND METHODS}

Young, recently expanded leaves were collected from one sample of the 10 patchouli accessions in the Active Germplasm Bank of Universidade Federal de Sergipe (Table 1). 
Table 1. Characteristics of the 10 patchouli accessions from the Active Germplasm Bank of Medicinal and Aromatic Plants of Universidade Federal de Sergipe.

\begin{tabular}{l|l|l|c}
\hline Accession code & Scientific name & Source & $\begin{array}{c}\text { No. in the Herbarium of } \\
\text { Universidade Federal de } \\
\text { Sergipe }\end{array}$ \\
\hline POG-001 & Pogostemon heyneanus & $\begin{array}{l}\text { Multidisciplinary Center for Chemical, Biological and } \\
\text { Agricultural Research, Universidade Estadual de } \\
\text { Campinas, Campinas, SP, Brazil }\end{array}$ & 13,169 \\
\hline POG-002 & Pogostemon cablin & Free market, São Paulo, SP, Brazil & 13,170 \\
\hline POG-006 & Pogostemon heyneanus & Richters company, Canada & 13,171 \\
\hline POG-014 & Pogostemon cablin & Free market, Belo Horizonte, MG, Brazil & 13,172 \\
\hline POG-015 & Pogostemon cablin & Free market, São Cristóvão, SE, Brazil & 13,173 \\
\hline POG-016 & Pogostemon cablin & Embrapa Genetic and Biotechnology Resources, Brasília, DF, Brazil & 13,174 \\
\hline POG-019 & Pogostemon cablin & Embrapa Genetic and Biotechnology Resources, Brasília, DF, Brazil & 13,175 \\
\hline POG-021 & Pogostemon cablin & Univeridade Federal do Paraná, Curitiba, PR, Brazil & 13,176 \\
\hline POG-022 & Pogostemon cablin & Growmore Ltd company, India & 13,177 \\
\hline
\end{tabular}

\section{Molecular analyses with SSR markers}

The cetyl trimethyl ammonium bromide (CTAB) protocol described by Doyle and Doyle (1990) was used to extract genomic DNA from the samples and a genomic library enriched for patchouli SSRs was generated (Billotte et al., 1999). The genomic DNA was digested with the enzyme Afal, and the digestion product was ligated to the adaptors Afa 21 (5'-CTCTTGCTTACGCGTGGACTA$\left.3^{\prime}\right)$ and Afa 25 (5'-TAGTCCACGCGTAAGCAAGAGCACA-3'). The fragments were pre-amplified using polymerase chain reaction (PCR) and purified with the QIAquick ${ }^{\circledR}$ PCR Purification kit (Qiagen, Chatsworth, CA, EUA) according to the manufacturer protocol.

Fragments containing SSRs were selected using beads (magnetic spheres) bound to streptavidin from the Streptavidin MagneSphere Paramagnetic Particles kit (Promega). Beads prewashed with purified DNA were mixed with the biotinylated SSR oligonucleotides (CT) and (GT).

The selected fragments were amplified using PCR, and the amplified fragments were

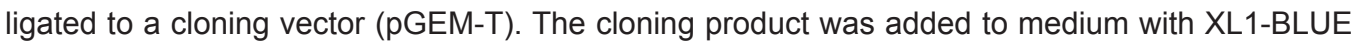
cells for bacterial transformation, and the insert was, thus, amplified. Colonies with a white color were replica-plated on an ELISA plate. The construct (vector + fragment) was maintained in U-bottom plates under appropriate conditions for subsequent analysis, and a step was performed to check if the transformed clones contained the inserts. The plasmid DNA was isolated from the recombinant colonies, and the insert was amplified so that the fragment could be sequenced. To eliminate possible interfering products or an excess of the sequencing reaction reagents, the samples were purified and sequenced.

WebSat (http://wsmartins.net/websat) was used to identify SSR regions and primers were designed with Primer3 software (Untergrasser et al., 2012). Gene Runner v. 3.05 (Hastings Software Inc., Hastings, NY, USA; http://www.generunner.net/) was used to validate the parameter values established for primer design and demonstrate the probability of formation of secondary structures preventing the use of the primers. Finally, the Chromas (Technelysium Pty Ltd., Australia; http://www.technelysium.com.au) program was used in the sequencing analysis of the SSR and primer regions.

The reaction was optimized on agarose gel, and the primers were tested on a polyacrylamide gel. For those primers that did not yield any bands, even with a temperature gradient, a touch down program was also used. The PCR-amplified fragments were subjected to $7 \%$ denaturing polyacrylamide gel electrophoresis and stained with silver nitrate. 
The 10 accessions were divided into two clusters based on the results obtained by Blank et al. (2011): one cluster consisted of POG-001 and POG-006 and the other cluster consisted of the remaining 8 accessions. In the statistical-genetic analyses, the allele frequency, observed heterozygosity $\left(H_{\mathrm{O}}\right)$, expected heterozygosity $\left(H_{\mathrm{E}}\right)$, allele amplitude, and polymorphic information content (PIC) were determined using MStools (Park, 2001). FSTAT provided the number of alleles per locus $\left(N_{\mathrm{A}}\right)$, allelic richness and $\mathrm{F}$-statistics (fixation index $\mathrm{F}_{\mathrm{ST}}$ and inbreeding coefficient $\mathrm{F}_{\mathrm{IS}}$ ) using Weir and Cockerham's methods (1984) (Goudet, 1995). The allele and genotype frequencies of each locus were calculated using the TFPGA program (Miller, 1997). The Structure software (Pritchard et al., 2000) was used to estimate the most probable number of clusters of samples. The number of clusters, K, was defined according to the criteria proposed by Evanno et al. (2005).

\section{Molecular analyses with ISSR markers}

Genomic DNA was extracted as described for SSR markers. After extraction, samples were quantified and diluted in Tris-EDTA buffer to a final concentration of $3 \mathrm{ng} / \mu \mathrm{L}$. PCR was optimized using accessions POG-001 and POG-014 to determine which primers would amplify DNA fragments with specific, polymorphic bands.

A total of 41 ISSR primers already available in the literature were tested, and 22 were selected. To determine the annealing temperature, a temperature gradient was tested, and the temperature showing the best amplification of non-specific, robust bands was selected.

PCR products were analyzed using $1.4 \%$ agarose gel electrophoresis and stained with blue green loading dye I (LGC Biotechnology). After the run, the gels were photographed under ultraviolet light, which revealed the DNA fragments marked with the dye. The bands for each primer were analyzed according to their presence (1) or absence (0).

Arlequin version 3.11 was used to perform analysis of molecular variance (AMOVA) (Excoffier et al., 2005). PopGene version 1.31 was used to calculate genetic similarities between the two clusters, assuming that the populations are in Hardy-Weinberg equilibrium (Yeh et al., 1999). The estimates used were $N_{A}$, effective number of alleles $\left(N_{E}\right)$ (Kimura and Crow, 1964), $H_{E}$ (Nei, 1973), Shannon index, and PIC.

The Structure software was used to estimate the number of clusters present for the studied patchouli accessions (Pritchard et al., 2000). DarWin was used to generate a dendrogram of the cluster formation, using Dice dissimilarity.

When evaluating chemical variations of the essential oil of the patchouli accessions in the Active Germplasm Bank of Universidade Federal de Sergipe (except POG-017), Blank et al. (2011) detected two clusters through multivariate analyses of four harvests, with POG-001 and POG-006 forming the first cluster ( $P$. heyneanus), and the remaining accessions forming the second cluster (P. cablin).

\section{RESULTS}

\section{Molecular analyses with SSR markers}

Sequencing of 96 samples from a plate using the genomic library revealed 24 SSR sequences, a $25 \%$ enrichment index. Of the SSR motifs identified, 10 were dinucleotides, four were trinucleotides, eight were tetranucleotides, one was a pentanucleotide, and one was a compound. Among the SSRs identified, only six pairs of primers were designed, making it necessary to sequence other colonies. 
The enrichment index in the second sequencing reaction (10.4\%) was lower than that in the first, and only 10 SSRs were identified among 96 samples. These motifs were classified and quantified as two dinucleotides, three trinucleotides, three tetranucleotides, one pentanucleotide, and one motif that was redundant with the first sequencing reaction. Six additional pairs of primers were designed from these nine non-redundant sequences.

A total of 12 pairs of primers were synthesized and named Pca1 to Pca12. Following optimization of the primers on a polyacrylamide gel, six polymorphic loci were used in further analyses (Table 2).

Table 2. Details of the six synthesized single sequence repeat (SSR) primer pairs used to characterize 10 patchouli accessions from the Active Germplasm Bank of Medicinal and Aromatic Plants Universidade Federal de Sergipe.

\begin{tabular}{l|c|l|c|c|l}
\hline Primer & Motif & Classification & Allele amplitude $(\mathrm{bp})$ & $\mathrm{T}\left({ }^{\circ} \mathrm{C}\right)$ & Primer sequence \\
\hline Pca 1 & GA(16) & Dinucleotide & $228-240$ & 57.2 & $\begin{array}{l}\text { F: } \text { ACACACTCCCCACCATAC } \\
\text { R: CCACCTGTTCTTTCACTTCC }\end{array}$ \\
\hline Pca 3 & CA(8) & Dinucleotide & $164-168$ & Touchdown & $\begin{array}{l}\text { F: CCATTCGTCACCTCTC } \\
\text { R: AAACAGGCAAGTGAAAGT }\end{array}$ \\
\hline Pca 4 & AG(14) & Dinucleotide & $258-266$ & 65 & $\begin{array}{l}\text { F: } \text { AGGGAAGCAGGGAAAACATT } \\
\text { R: CTTGCGGCATTTAGGGATAC }\end{array}$ \\
\hline Pca 6 & TG(4).TC.TG(5)AG(4)...TGTT(3) & Compound & $180-216$ & Touchdown & $\begin{array}{l}\text { F: ACAAAGGGTTGACGATTG } \\
\text { R: GTGATGAAACTGTCTCTCCTG }\end{array}$ \\
\hline Pca 7 & TG(6).AGTC.TA(4) & Compound & $186-220$ & 57.2 & $\begin{array}{l}\text { F: } \text { AAGCGTGATTGCCCTTAAT } \\
\text { R: GCCGTATTTGAGAATGCTT }\end{array}$ \\
\hline Pca 8 & CAT(5) & Trinucleotide & $207-210$ & Touchdown & $\begin{array}{l}\text { F }: \text { ACCTGACGCCACTACTCCTC } \\
\text { R: TCACTTTGAATGCGACCA }\end{array}$ \\
\hline
\end{tabular}

Using the optimized primers, 14 alleles were identified for the six loci studied. The Pca1 primer revealed the highest number of alleles (4), whereas all the other loci revealed two alleles, with an average of 2.33 alleles per locus.

Of the polymorphic microsatellite loci, five contained dinucleotide motifs, and only one featured a trinucleotide motif. All the primers designed for tetranucleotide or pentanucleotide microsatellites were monomorphic.

For the Pca1 locus, $H_{\mathrm{E}}$ was 0.66 for cluster $A$ and 0.53 for cluster $\mathrm{B}$, whereas $H_{\mathrm{O}}$ was 1 for both clusters at the same locus. For the other loci, $H_{E}$ and $H_{\mathrm{O}}$ were both 0 . The PIC value for Pca1 was 0.375 for both clusters studied. For the other primers, PIC was 0 .

Using the matrix of genetic distances created by Rogers (1972), modified by Wright (1978), and according to the Neighbor-Joining method clustering criteria, a dendrogram was generated using the TFPGA program (Miller, 1997), which shows the relationships between the 10 accessions studied (Figure 1).

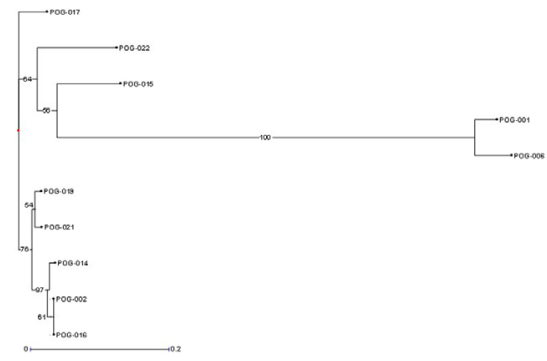

Figure 1. Dendrogram of 10 patchouli accessions from the Active Germplasm Bank of Universidade Federal de Sergipe based on single sequence repeat (SSR) loci, created using the UPGMA method and Roger's genetic distance. Numbers on branches indicate confidence from 1000 bootstrap repetitions. 
The Structure software proved to be an efficient tool in cluster identification because it was successful in clustering the 10 accessions into two distinct, well-defined clusters: the black colored cluster, containing POG-001 and POG-006 (P. heyneanus), and the gray colored cluster, containing the remaining accessions ( $P$. cablin) (Figure 2A).

A

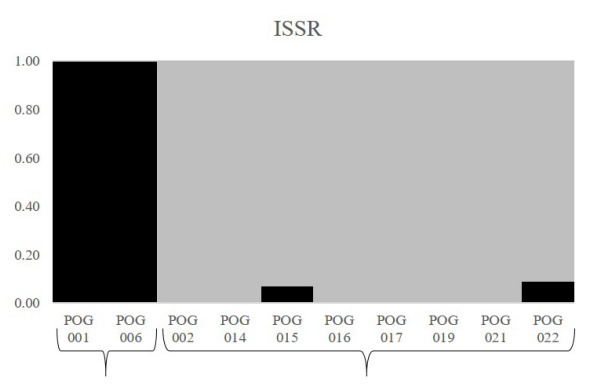

P. heyneanus

\begin{abstract}
P. cablin
\end{abstract}
$\mathrm{B}$

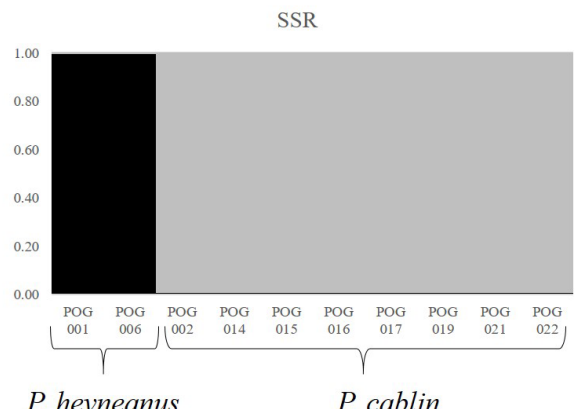

Figure 2. Subdivision of 10 patchouli accessions from the Active Germplasm Bank of Universidade Federal de Sergipe into clusters based on inter-simple sequence repeat (ISSR) data (A) and single sequence repeat (SSR) data (B), inferred from Bayesian analysis performed using the Structure program $(K=2)$. Each accession is represented by a vertical bar, with bars of the same color clustered together. Membership of each accession to each cluster is shown on the y-axis.

\section{Molecular analyses with ISSR markers}

Of the 41 primers used, 22 generated specific bands. Of the 181 bands obtained, a total of 146 were polymorphic $(80.66 \%)$ and 35 were monomorphic, with an average of 8.23 bands per primer. The number of bands identified per primer ranged from 3 (primer 7) to 20 (primer MAO; Table 3).

Table 3. Details of the 22 inter simple sequence repeat (ISSR) primers that successfully amplified specific bands in 10 patchouli accessions from the Active Germplasm Bank of Medicinal and Aromatic Plants of Universidade Federal de Sergipe.

\begin{tabular}{|c|c|c|c|c|c|}
\hline Primer name & Sequence & Annealing temperature $\left({ }^{\circ} \mathrm{C}\right)$ & No. of bands & No. of polymorphic bands & No. of monomorphic bands \\
\hline JOHN & $(\mathrm{AG})_{7} \mathrm{CC}$ & 45 & 4 & 4 & 0 \\
\hline 843 & $(\mathrm{CT})_{8} \mathrm{AA}$ & 47.8 & 7 & 4 & 3 \\
\hline 844 & $(\mathrm{CT})_{8} \mathrm{AC}$ & 45 & 12 & 11 & 1 \\
\hline 898 & $(\mathrm{CA})_{6} \mathrm{AT}$ & 32 & 13 & 10 & 3 \\
\hline 899 & (CA) $)_{6} \mathrm{AG}$ & 33.8 & 7 & 4 & 3 \\
\hline 902 & $(\mathrm{GT})_{6} \mathrm{AT}$ & 45 & 4 & 3 & 1 \\
\hline AW3 & $(\mathrm{GT})_{6} \mathrm{GG}$ & 45 & 4 & 4 & 0 \\
\hline MANNY & $(\mathrm{CAC})_{4} \mathrm{AC}$ & 45 & 11 & 11 & 0 \\
\hline GOOFY & $(\mathrm{GT})_{7} \mathrm{CC}$ & 52 & 7 & 6 & 1 \\
\hline TERRY & $(\mathrm{GTC})_{4} \mathrm{GC}$ & 45 & 5 & 4 & 1 \\
\hline BECKY & $(\mathrm{CA})_{7} \mathrm{TC}$ & 50 & 10 & 9 & 1 \\
\hline MAO & $(\mathrm{CTC})_{4} \mathrm{AC}$ & 45 & 20 & 19 & 1 \\
\hline OMAR & $(\mathrm{GAG})_{4} \mathrm{GC}$ & 48 & 7 & 4 & 3 \\
\hline DAT & $(\mathrm{GA}) \rightarrow \mathrm{AG}$ & 45 & 16 & 12 & 4 \\
\hline 1 & $(\mathrm{AG})_{9}$ & 54 & 6 & 3 & 3 \\
\hline 5 & $(\mathrm{GAG})_{5}$ & 54 & 7 & 3 & 4 \\
\hline 7 & $(\mathrm{CT})_{8} \mathrm{AG}$ & 56.2 & 4 & 2 & 1 \\
\hline 7 & $(\mathrm{GTG})_{5}$ & 52 & 3 & 3 & 1 \\
\hline 18 & $(\mathrm{GA})_{9} \mathrm{~T}$ & 59 & 8 & 8 & 0 \\
\hline ISSR3 & $(\mathrm{CTC})_{4} \mathrm{RC}$ & 45 & 11 & 9 & 2 \\
\hline ISSR4 & $(\mathrm{AG})_{8} \mathrm{YC}$ & 48 & 8 & 7 & 1 \\
\hline ISSR6 & $(\mathrm{CA})_{8} \mathrm{G}$ & 48 & 7 & 6 & 1 \\
\hline
\end{tabular}

ISSR data also shows high $\mathrm{F}_{\mathrm{ST}}$ value (0.905) between Pogostemon heyneanus and Pogostemon cablin clusters. 
The mean $N_{\mathrm{A}}, N_{\mathrm{E}}$, Nei's (1973) gene diversity, and Shannon's Information index were $1.055,1.055,0.028$, and 0.038 , respectively, for $P$. heyneanus and 1.210, 1.089, 0.059, and 0.095, respectively, for $P$. cablin (Table 4). The average Nei's gene diversity (1973) and the Shannon index (Lewontin, 1972) were 0.0229 and 0.0334 , respectively, for $P$. heyneanus and 0.0695 and 0.1046 , respectively, for $P$. cablin. The percentage of polymorphic loci was $5.52 \%$ for the first cluster and $20.99 \%$ for the second cluster.

Table 4. Genetic parameters of the patchouli accessions from the Active Germplasm Bank of Universidade Federal de Sergipe based on inter-simple sequence repeat (ISSR) data.

\begin{tabular}{l|c|c|c|c}
\hline & $N_{\mathrm{A}}$ & $N_{\mathrm{E}}$ & $\mathrm{h}$ & $\mathrm{I}$ \\
\hline Pogostemon heyneanus & $1.055(0.229)$ & $1.055(0.229)$ & $0.028(0.115)$ & $0.038(0.159)$ \\
\hline Pogostemon cablin & $1.210(0.408)$ & $1.089(0.243)$ & $0.059(0.124)$ & $0.095(0.191)$ \\
\hline Overall & $1.807(0.396)$ & $1.399(0.243)$ & $0.261(0.142)$ & $0.405(0.212)$ \\
\hline
\end{tabular}

$N_{\mathrm{A}}$, observed number of alleles; $N_{\mathrm{E}}$, effective number of alleles (Kimura and Crow, 1964); h, Nei's (1973) gene diversity; I, Shannon's Information index (Lewontin, 1972). Standard deviations are show in parentheses.

AMOVA for the 10 patchouli accessions studied showed higher genetic variation between the two clusters $(90.51 \%)$ than within the clusters $(9.49 \%$; Table 5$)$.

Table 5. Analysis of molecular variance (AMOVA) for the patchouli accessions from the Active Germplasm Bank of Medicinal and Aromatic Plants of Universidade Federal de Sergipe based on inter simple sequence repeat (ISSR) data.

\begin{tabular}{l|c|c|c|c}
\hline Variation source & Degrees of freedom & Sum of squares & $\begin{array}{c}\text { Variance } \\
\text { components }\end{array}$ & Variation percentage \\
\hline Between populations & 1 & 188.625 & $57.07520 \mathrm{Va}$ & 90.51 \\
\hline Within populations & 8 & 47.875 & $5.98438 \mathrm{Vb}$ & 9.49 \\
\hline Total & 9 & 236.500 & 63.05957 & \\
\hline FST & \multicolumn{3}{|c}{0.90510} \\
\hline
\end{tabular}

The use of the Structure program led to the formation of two specific clusters from the 10 accessions studied. One of the clusters consisted of accessions POG-001 and POG-006. All the remaining patchouli accessions of the Active Germplasm Bank Universidade Federal de Sergipe formed the second cluster, colored in black (Figure 2B).

DarWin was used to identify the individual clusters, placing accessions POG-001 and POG-006 in a cluster separate from that of the other accessions (Figure 3).

The $F_{\mathrm{ST}}$ calculated $(0.833)$ shows the divergence between $P$. heyneanus and $P$. cablin clusters. Deficit of heterozygotes was detected by $F_{\text {IS }}$ estimate $(0.917)$ (Table 6). 


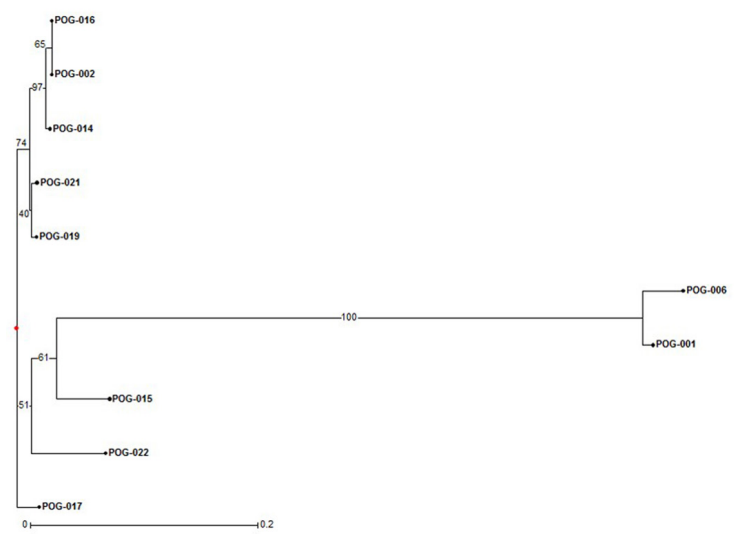

Figure 3. Dendrogram of 10 patchouli accessions from the Active Germplasm Bank of Universidade Federal de Sergipe based on Dice genetic dissimilarity from inter-simple sequence repeat (ISSR) loci, created using the UPGMA method. Numbers on branches indicate confidence from 1000 bootstrap repetitions.

Table 6. Weir and Cockerham (1984) estimation of $F_{\mathrm{ST}}$ (theta) and $F_{\text {IS }}(\mathrm{f})$ for the patchouli accessions from the Active Germplasm Bank of Medicinal and Aromatic Plants of Universidade Federal de Sergipe based on SSR data.

\begin{tabular}{l|c|c} 
& Theta & $\mathrm{f}$ \\
\hline Main & 0.833 & 0.917 \\
\hline Lower Cl & 0.75 & -1 \\
\hline Upper Cl & 1 & 1 \\
\hline
\end{tabular}

$\mathrm{Cl}$, confidence interval.

\section{DISCUSSION}

\section{SSRs}

The use of molecular markers is an efficient option for the characterization of germplasm banks, which is necessary for appropriate maintenance of biological resources to ensure no genetic losses or duplications occur. The division of the patchouli accessions into two clusters was based on a study by Blank et al. (2011). Considering that POG-001 and POG-006 are different species of $P$. cablin, the genetic divergence between individuals - if placed into a single cluster - would be very high, but would not represent the real genetic characterization of the accessions.

One of the advantages of using SSR markers is their codominance, allowing individuals with heterozygous loci to be differentiated from homozygous individuals. In this study, only POG001 and POG-006 featured heterozygous loci, which may be explained by the fact that they are the only plants among the 10 accessions that generate inflorescences in northeast Brazil. It is likely that, in their native environment, there was gamete exchange between plants, which enabled genetic recombination and heterozygote emergence. Because the other genotypes of patchouli from the Active Germplasm Bank of Universidade Federal de Sergipe do not produce flowers and reproduce asexually, there has been no allele exchange between different individuals. In general, in their natural habitat, heterozygous plants of $P$. cablin must be uncommon. 
In both the first and second sequencing reactions, primers were not designed for all SSR sequences identified because it was not possible to design good quality primers. This occurred due to the proximity of SSRs to the adapters or because the flanking regions of the fragments did not have good sequencing quality, preventing primer design.

Among the polymorphic SSR loci, five contained dinucleotide motifs and only one featured a trinucleotide motif. All the primers designed for SSRs with tetranucleotide or pentanucleotide motifs were monomorphic. The dinucleotide motifs were polymorphic because they had more repetitions than, for example, the pentanucleotides. This finding has already been reported by Chin et al. (1996), who determined that dinucleotide microsatellite repetitions are frequently the most polymorphic class, and by Zhu et al. (2003), who correlated the non-occurrence of polymorphisms in microsatellites with few repetitions in tandem with the "slippage" of the DNA polymerase. Therefore, loci with a higher number of repetitions are more susceptible to mutation, and there is a higher probability of error by the DNA polymerase during DNA replication through slippage. Strassmann et al. (1997) explained that it is expected that loci with fewer than five repetitions are rarely polymorphic, as in the case for patchouli.

In general, the SSR motifs more commonly found in plant genomes are the AT/TA dinucleotides, followed by AA/TT and GA/CT (Pinto et al., 2001). More than half the SSR loci used in this study featured these repetitions, which confirm the data by Pinto et al. (2001).

For the Pca1 locus, because $H_{\mathrm{E}}$ was lower than $H_{\mathrm{O}}$ in both clusters, we concluded that there is an excess of heterozygotes relative to the expected number based on Hardy-Weinberg equilibrium. de Moura et al. (2009) also found a higher $H_{\mathrm{O}}$ than $H_{E}$ in a study of Solanum lycocarpum, which suggests an excess of heterozygotes. The observed heterozygosity may be considered a relationship between the heterozygous individuals in the cluster.

A higher average index of $H_{\mathrm{O}}$ and $H_{\mathrm{E}}$ relative to other studies may result in part from the lower number of individuals evaluated here. A sampling error based on the small sample size used here could lead to a lack of possible representative genotypes for each locus. The presence of a higher number of homozygotes in the studied cluster, with heterozygotes being rare, may be explained by the fact that patchouli reproduction occurs via cuttings. Therefore, in future study, population heterozygosity data will probably be in agreement with those of other researchers if a larger sample of individuals is used.

Regarding the other loci studied, $H_{\mathrm{E}}$ and $H_{\mathrm{O}}$ were null, indicating a deficit of heterozygotes. According to Huang et al. (2009), this could suggest a loss of genetic polymorphism due to genetic drift or possibly due to population structuring within that cluster. These two hypotheses could explain the null $H_{\mathrm{E}}$ and $H_{\mathrm{O}}$, and they also imply that individuals in each cluster may be clones.

Analyzing the plots of allele frequencies, it is evident that for the primers studied, the alleles of the first cluster are not shared with any individual in the second cluster and vice-versa.

P. cablin alleles (POG-002, POG-014, POG-015, POG-016, POG-017, POG-019, POG021, and POG-022) are shared by all the accessions in this cluster. The same is true for Pogostemon sp (POG-001, POG-006) accessions evaluated for their alleles.

A good indicator of marker quality in genetic studies is PIC, described by Botstein et al. (1980). According to the classification by this author, markers with PIC values higher than 0.5 are considered to be very informative, those with values between 0.25 and 0.50 are moderately informative, and those with values lower than 0.25 have little informative value. According to this classification, only Pca1 was a moderately informative marker because its PIC value was 0.375 , whereas the other markers were not informative, with PIC values of 0 . However, this low value, which tends to suggest a marker of low quality in terms of its informativeness, is due to the low number of 
individuals in the study; if we increase the sample size, the PIC value will probably change.

The Bayesian analysis performed using the Structure software confirmed that there is no allele sharing between the accessions in the first cluster relative to the second cluster and vice-versa, at least for the primers used in this study. The graph produced by Structure showed filled vertical bars, representing total certainty that the accessions were grouped correctly. The dendrogram obtained with the Neighbor-joining method also showed the formation of two well-specified clusters, which correspond to the results of the Structure analysis. From these analyses, it was determined that the Pogostemon sp accessions are genetically very distant from the $P$. cablin accessions.

\section{ISSRs}

While ISSR markers are also dominant, they are more reproducible than random amplified polymorphic DNA. This reproducibility is associated with two characteristics that provide higher specificity to the ISSR primers, their higher annealing temperature and their design based on microsatellite regions, making them semi-arbitrary markers.

A high number of primers yielding polymorphic, robust bands were obtained in this study (22), considering that in several other research studies, the number of primers used was approximately 10. de Almeida et al. (2009), who performed molecular characterization of sugarcane with ISSR markers, were able to generate robust bands with eight primers. Hussein et al. (2008) used nine primers to amplify fragments in studies of strawberry. From 21 initial primers, Djè et al. (2006) obtained bands with good amplification using 11 of the primers in studies of Cucurbitaceae.

The number of bands obtained from the 22 primers was relatively high ( 146 polymorphic) and was close to that reported by Wu et al. (2010) in a study of $P$. cablin populations using ISSR markers, who observed a total of 241 bands from 19 primers. The percentage of polymorphic bands relative to all accessions was very close to the results of Pan et al. (2006), who genetically characterized five $P$. cablin populations using RAPD markers, identifying $79.76 \%$ of the total bands as polymorphic, which enabled the identification of genetic variation within the different populations studied.

The Shannon index is a metric that ranges from 0 to 1 ; the closer that value is to 0 , the lower the diversity. This index was close to 0 for the two clusters studied here, establishing that the diversity within these clusters is low. These results were expected upon visual analysis of the agarose gel because it was evident that almost no variation in terms of the height of the bands existed within the clusters studied.

The $F_{\text {ST }}$ value $(0.90510)$, which was obtained from AMOVA, was highly significant $(\mathrm{P}<$ 0.001 ). This value indicates that $9.49 \%$ of the total variation occurs within the clusters and that $90.51 \%$ occurs between the clusters. This high value $\left(F_{\mathrm{ST}}=0.9051\right)$ may indicate that there is reduced gene flow between the clusters, leading to an increase in genetic differentiation. According to Hartl and Clark (2007), $F_{\mathrm{ST}}$ values above 0.25 indicate very high genetic differentiation. We suggest that the high genetic variation between the two clusters, provided by AMOVA, probably occurred because the $P$. heyneanus individuals belong to a different species from that of $P$. cablin cluster.

The low genetic diversity found within each cluster studied in this study may be associated with the fact that patchouli is not a species native to Brazil and is present in a germplasm bank, without allele exchange between individuals. Moreover, according to Paul et al. (2010), the propagation of patchouli occurs via cuttings, and there is no allele exchange through sexual reproduction.

The use of the two types of molecular markers evaluated in this article enabled a more general analysis of the active germplasm bank studied. The specificity of the SSR markers proved to be efficient for differentiation of the accessions into two well-defined clusters. To compensate 
for the limited number of individuals, characterization with ISSR markers was necessary and completed the analysis satisfactorily. Being semi-arbitrary markers, ISSRs enabled the detection of a higher number of bands and also confirmed the results obtained with the SSRs for Pogostemon sp.

Based on these results, we conclude that POG-001 and POG-006 are different species of $P$. cablin, commonly known by the same name (patchouli) and classified as $P$. heyneanus by the herbarium of Universidade Federal de Sergipe. The analysis using the six pairs of SSR primers placed the other individuals from the active germplasm bank into a single cluster, as clones.

The SSR primers described here were very efficient in the detection of polymorphisms because we obtained polymorphic bands. The results obtained with ISSR markers indicate that these markers may be used efficiently in the fingerprinting of patchouli accessions.

\section{Conflicts of interest}

The authors declare no conflict of interest.

\section{ACKNOWLEDGMENTS}

The authors thank CAPES, CNPq, FAPITEC/SE, and FINEP for their financial support of this study.

\section{REFERENCES}

Blank AF, Sant'ana TCP, Santos PS, Arrigoni-Blank MF, et al. (2011). Chemical characterization of the essential oil from patchouli accessions harvested over four seasons. Ind. Crops Prod. 34: 831-837. http://dx.doi.org/10.1016/j.indcrop.2011.01.021

Billotte N, Lagoda PJL, Risterucci AM and Baurens FC (1999). Microsatellite-enriched libraries: applied methodology for the development of SSR markers in tropical crops. Fruits 54: 277-288.

Botstein D, White RL, Skolnick M and Davis RW (1980). Construction of a genetic linkage map in man using restriction fragment length polymorphisms. Am. J. Hum. Genet. 32: 314-331.

Chin L, Liégeois N, DePinho RA and Schreiber-Agus N (1996). Functional interactions among members of the Myc superfamily and potential relevance to cutaneous growth and development. J. Investig. Dermatol. Symp. Proc. 1: 128-135.

de Almeida CMA, de Lima SEN, de Andrade Lima GS, de Brito JZ, et al. (2009). Caracterização molecular de cultivares de cana-de-açúcar utilizando marcadores ISSR. Cienc. Agrotec. 33: 1771-1776. http://dx.doi.org/10.1590/S1413$\underline{70542009000700012}$

de Moura TM, Sebbenn AM, Chaves LJ, Coelho ASG, et al. (2009). Diversidade e estrutura genética espacial em populações fragmentadas de Solanum spp do Cerrado, estimadas por meio de locos microssatélites. Sci. Forum 37: $143-150$.

Djè Y, Tahi GC, Zoro Bi IA, Malice M, et al. (2006). Optimization of ISSR marker for African edible-seeded Cucurbitaceae species' genetic diversity analysis. Afr. J. Biotechnol. 5: 83-87.

Doyle JJ and Doyle JL (1990). Isolation of plant DNA from fresh tissue. Focus 12: 13-15.

Evanno G, Regnaut S and Goudet J (2005). Detecting the number of clusters of individuals using the software STRUCTURE: a simulation study. Mol. Ecol. 14: 2611-2620. http://dx.doi.org/10.1111/j.1365-294X.2005.02553.x

Excoffier L, Laval G and Schneider S (2005). Arlequin (version 3.0): an integrated software package for population genetics data analysis. Evol. Bioinform. Online 1: 47-50.

Goudet J (1995). FSTAT (version 1.2): a computer program to calculate F-statistics. J. Hered. 86: 485-486.

Hartl DL and Clark AG (2007). Principles of population genetics. Sinauer Associates, Sunderland.

Hsu HC, Yang WC, Tsai WJ, Chen CC, et al. (2006). Alpha-bulnesene, a novel PAF receptor antagonist isolated from Pogostemon cablin. Biochem. Biophys. Res. Commun. 345: 1033-1038. http://dx.doi.org/10.1016/j.bbrc.2006.05.006

Huang HR, Zhou G, Ge XJ, Wei X, et al. (2009). Eight polymorphic microsatellite loci for the Chinese medicinal plant Artemisia annua L. (Asteraceae). Conserv. Genet. 10: 593-595. http://dx.doi.org/10.1007/s10592-008-9585-2

Hussein TS, Tawfik AA and Khalifa MA (2008). Molecular identification and genetic relationships of six strawberry varieties using ISSR markers. Int. J. Agric. Biol. 10: 677-680. 
Kelkar YD, Strubczewski N, Hile SE, Chiaromonte F, et al. (2010). What is a microsatellite: a computational and experimental definition based upon repeat mutational behavior at A/T and GT/AC repeats. Genome Biol. Evol. 2: 620-635. http://dx.doi. org/10.1093/gbe/evq046

Kimura M and Crow JF (1964). The number of alleles that can be maintained in finite population. Genetics 49: $725-738$.

Lewontin RC (1972). Testing the theory of natural selection. Nature 236: 181-182. http://dx.doi.org/10.1038/236181a0

Liu B and Wendel JF (2001). Intersimple sequence repeat (ISSR) polymorphisms as a genetic marker system in cotton. Mol. Ecol. Notes 1: 205-208. http://dx.doi.org/10.1046/j.1471-8278.2001.00073.x

Miller MP (1997). Tools for population genetics analyses (TFPGA) version 1.3. A Windows program for the analysis of allozyme and molecular population genetic data. Department of Biological Sciences, Northern Arizona University, Flagstaff, Arizona.

Murugan R and Livingstone C (2010). Origin of the name 'patchouli' and its history. Curr. Sci. 99: 1274-1276.

Nei M (1973). Analysis of gene diversity in subdivided populations. Proc. Natl. Acad. Sci. USA 70: 3321-3323. http://dx.doi. org/10.1073/pnas.70.12.3321

Oyen LPA (1999). Pogostemon Desf. L. In: Plant resources of south-east Asia No 19: essential-oil plants (Oyen LPA and Dung NX, eds). Backhuys Publishers, 151-157.

Pan CM, Li W, He H, Deng WQ, et al. (2006). [Study on intraspecific genetic diversity in different plant populations of Pogostemon cablin]. Zhongguo Zhong Yao Za Zhi 31: 723-726.

Park S (2001). MStools v 3 (Excel spreadsheet toolkit for data conversion). Smurfit Institute of Genetics, Trinity College, Dublin 2.

Paul A, Thapa G, Basu A, Mazumdar P, et al. (2010). Rapid plant regeneration, analysis of genetic fidelity and essential aromatic oil content of micropropagated plants of Patchouli, Pogostemon cablin (Blanco) Benth. An industrially important aromatic plant. Ind. Crops Prod. 32: 366-374. http://dx.doi.org/10.1016/j.indcrop.2010.05.020

Pinto LS, Vieira MLC, Souza AP and Souza Júnior CL (2001). Isoenzimas e microssatélites em plantas. Biotecnolog. Cienc. Desenvolv. 20: 16-19.

Pritchard JK, Stephens M and Donnelly P (2000). Inference of population structure using multilocus genotype data. Genetics 155: 945-959.

Remya KS, Joseph S, Lakshmi PK and Akhila S (2010). Microsatellites in varied arenas of research. J. Pharm. Bioallied Sci. 2: 141-143. http://dx.doi.org/10.4103/0975-7406.67004

Rogers JS (1972). Measures of genetic similarity and genetic distances. Stud. Gen. (Berl.) 7213: 145-153.

Singh M and Rao RSG (2009). Influence of sources and doses of N and K on herbage, oil yield and nutrient uptake of patchouli [Pogostemon cablin (Blanco) Benth.] in semi-arid tropics. Ind. Crops Prod. 29: 229-234. http://dx.doi.org/10.1016/j. indcrop.2008.05.005

Strassmann JE, Barefield K, Solís CR, Hughes CR, et al. (1997). Trinucleotide microsatellite loci for a social wasp, Polistes. Mol. Ecol. 6: 97-100. http://dx.doi.org/10.1046/j.1365-294X.1997.00158.x

Sugimura Y, Kadotani N, Ueda Y, Shima K, et al. (2005). Transgenic patchouli plants produced by Agrobacterium-mediated transformation. Plant Cell Tissue Organ Cult. 82: 251-257. http://dx.doi.org/10.1007/s11240-005-1039-7

Swamy MK, Balasubramanya S and Anuradha M (2010). In vitro multiplication of Pogostemon cablin Benth. through direct regeneration. Afr. J. Biotechnol. 9: 2069-2075.

Untergasser A, Cutcutache I, Koressaar T, Ye J, et al. (2012). Primer3--new capabilities and interfaces. Nucleic Acids Res. 40: e115. http://dx.doi.org/10.1093/nar/gks596

Weir BS and Cockerham CC (1984). Estimating F-statistics for the analysis of population structure. Evolution 38: $1358-1370$. http://dx.doi.org/10.2307/2408641

Wright S (1978). Evolution and the genetics of populations, Volume 4: variability within and among natural populations. University of Chicago Press, Chicago.

Wu YG, Guo QS, He JC, Lin YF, et al. (2010). Genetic diversity analysis among and within populations of Pogostemon cablin from China with ISSR and SRAP markers. Biochem. Syst. Ecol. 38: 63-72. http://dx.doi.org/10.1016/j.bse.2009.12.006

Xu X, Tang Z and Liang Y (2010). Comparative analysis of plant essential oils by GC-MS coupled with integrated chemometric resolution methods. Anal. Methods 2: 356-367. http://dx.doi.org/10.1039/b9ay00213h

Yeh FC, Yang RC and Boyle T (1999). PopGene Version 131: Microsoft Window-based freeware for population genetic analysis. University of Alberta and Centre for International Forestry Research.

Zietkiewicz E, Rafalski A and Labuda D (1994). Genome fingerprinting by simple sequence repeat (SSR)-anchored polymerase chain reaction amplification. Genomics 20: 176-183. http://dx.doi.org/10.1006/geno.1994.1151

Zhu BC, Henderson G, Yu Y and Laine RA (2003). Toxicity and repellency of patchouli oil and patchouli alcohol against Formosan subterranean termites Coptotermes formosanus Shiraki (Isoptera: Rhinotermitidae). J. Agric. Food Chem. 51: 4585-4588. http://dx.doi.org/10.1021/jf0301495 